\title{
The influence of the internal structuring of coronal loops on the properties of their damped transverse oscillations
}

\author{
I. Arregui, J. Terradas ${ }^{\star}$, R. Oliver, and J. L. Ballester
}

\author{
Departament de Física, Universitat de les Illes Balears, 07122 Palma de Mallorca, Spain \\ e-mail: [inigo.arregui; jaume.terradas; ramon.oliver; dfsjlbo]@uib.es \\ Received 22 November 2006 / Accepted 31 January 2007
}

\section{ABSTRACT}

\begin{abstract}
Context. The geometry and physical conditions in solar coronal loops are complicated and still not understood well. Recent highresolution observations obtained with TRACE indicate the existence of sub-resolution transverse structuring not accessible to direct observation. This ingredient has not yet been taken into account in the previous theoretical models used for the study of transversal coronal loop oscillations and of their damping due to resonant conversion of energy.

Aims. This study aims to assess the effect of the possibly unresolved internal structure of a coronal loop on the properties of its transverse oscillations and on the efficiency of resonant absorption as a damping mechanism of these oscillations.

Methods. The equilibrium configuration of a single coronal loop with internal density structuring is modelled by considering the loop as composed of two very close, parallel, identical coronal slabs in Cartesian geometry. The period of the oscillation and the damping time are computed for the resonantly damped fundamental kink mode. These quantities are then compared to those obtained for two models for a single equivalent slab without internal density structuring.

Results. We find that the period and the damping time of a coronal loop with internal density structuring change by less than $15 \%$, when compared to the same oscillatory properties of a single coronal loop with either the same density contrast or a single coronal loop with the same total mass.

Conclusions. Therefore the internal density structuring of a coronal loop does not affect its oscillatory properties very much. However, the sub-resolution structuring of a coronal loop with different densities in its components or with different widths could vary these results.
\end{abstract}

Key words. Sun: corona - Sun: magnetic fields - Sun: oscillations

\section{Introduction}

Among the different types of waves and oscillations detected in magnetic structures of the solar corona (see Nakariakov \& Verwichte 2005; Aschwanden 2006, for recent reviews), flareinduced transverse coronal loop oscillations have attracted particular interest since their first unambiguous detection with highresolution instruments on-board space-crafts, such as SoHO and TRACE (Aschwanden et al. 1999; Nakariakov et al. 1999). Measurements of physical and geometrical properties of these oscillations have been provided by Aschwanden et al. (2002) and Schrijver et al. (2002), while from the theoretical point of view, the observed oscillations have been interpreted in terms of fast magnetohydrodynamic (MHD) kink mode oscillations of a cylindrical flux tube in their fundamental harmonic (Nakariakov et al. 1999). This interpretation has opened the way to the novel discipline of coronal seismology, which is the determination of unknown physical parameters in the solar corona by comparing the observed and theoretical properties of waves and oscillations in magnetic structures, such as first suggested on theoretical grounds by Uchida (1970) and Roberts et al. (1984). Some recent examples of the application of coronal seismology can be found in Nakariakov \& Ofman (2001), Verwichte et al. (2006), and Arregui et al. (2007).

An important property of the observed oscillations is their rapid damping, with an exponential decay time of only a few

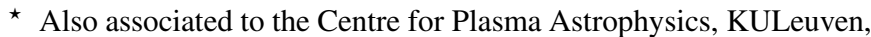
Celestijnenlaan 200B, 3001 Heverlee, Belgium. periods. Several theoretical mechanisms have been proposed to explain this damping: non-ideal effects, lateral wave leakage due to the curvature of the loops (Verwichte et al. 2006), mechanisms based on the topology of the magnetic field lines (Schrijver \& Brown 2000), and resonant conversion of wave energy from the global large scale kink eigenmode to localised Alfvén motions, due to the non-uniformity of the magnetic-plasma configuration (Hollweg \& Yang 1988; Ruderman \& Roberts 2002; Goossens et al. 2002, 2006). Foot-point leakage through the chromospheric density gradient (De Pontieu et al. 2001; Ofman 2002) has been invoked for the damping of Alfvén waves and could be ascribed to the damping of transverse oscillations, as well.

The geometry and physical conditions in real coronal loops are complicated and still not well-understood. Slender overdense coronal flux tubes have been commonly modelled by means of cylindrically symmetric flux tubes (Edwin \& Roberts 1983; Roberts 1983). More recently, theoretical models have explored second-order effects, such as the curvature of coronal loops (Van Doorsselaere et al. 2004; Terradas et al. 2006), the non-circularity of their cross-sections (Ruderman 2003), or the influence of a longitudinally varying density (Andries et al. 2005a,b; Arregui et al. 2005). Most of these previous models assume monolithic equilibrium configurations with uniform internal density in the direction transverse to field lines, which is an over-simplification of the real physical conditions in coronal loops. For instance, high-resolution observations suggest the existence of sub-resolution transverse structuring, not accessible to direct observation with our current capabilities 
(Schmelz et al. 2005). This view has been challenged in some works (Aschwanden \& Nightingale 2005; Aschwanden 2005), although it is supported in some others (e.g. Martens et al. 2002; Schmelz et al. 2003), so there is no definitive answer to this controversy yet.

The purpose of this work is to assess the effect of the possibly unresolved internal structure of a coronal loop on the properties of its transverse oscillations. To this end, a single coronal loop with transverse density structuring is modelled as being composed by a system of two coronal slabs. The slab geometry adopted in this work is far from being an accurate representation of the flux tube structures that form coronal loops. However, this simplification allows a more detailed study of the effects of internal structuring and its causes. The period, damping time, and damping rate of resonantly damped MHD fast modes are computed and these quantities are then compared to those corresponding to two other similar physical coronal slab models with identical widths and a uniform internal density and either with identical total mass or density contrast. The differences that arise from considering one or the other kind of system are established and the relative importance of the internal and external non-uniform layers of the full two-slab system is investigated.

The layout of the paper is as follows. In Sect. 2, the equilibrium configuration used for the modelling of a single coronal loop with internal density structuring is described. Also, the relevant linear MHD wave equations and the numerical procedure followed in our analysis are presented. Section 3 describes the analysis and the obtained results. Finally, in Sect. 4, our conclusions are drawn.

\section{Physical models and linear MHD waves}

\subsection{Equilibrium configuration}

We model the equilibrium magnetic and plasma configuration of a single coronal loop by means of a one-dimensional system of two identical parallel over-dense slabs in Cartesian geometry. The magnetic field is straight and pointing in the $z$-direction, $\boldsymbol{B}=B \hat{\boldsymbol{e}}_{z}$. For applications to the solar corona, it is a good approximation to consider that the magnetic pressure dominates the gas pressure. This classic plasma- $\beta=0$ approximation implies that the magnetic field is uniform and that the density $\rho(x)$ or Alfvén speed $v_{\mathrm{A}}(x)$ profiles can be chosen arbitrarily. The system of two coronal slabs is then modelled through a varying equilibrium density profile in the $x$-direction (see Fig. 1) by means of two density enhancements of half-width $a$ centred on $\pm x_{0}$, hence, the width of the simulated structured loop is $2 a_{\mathrm{s}}$, with $a_{\mathrm{s}}=a+x_{0}$. The density of each of the slabs is constant, $\rho_{\mathrm{i}}$, and connected to the constant coronal environment with density $\rho_{\mathrm{e}}$, by transitional non-uniform layers of thickness $l$. The explicit expression for the considered equilibrium density is

$$
\rho(x)=\left\{\begin{array}{lr}
\rho_{\mathrm{e}} & 0 \leq x \leq x_{0}-a-\frac{l}{2}, \\
f_{1}(x) & x_{0}-a-\frac{l}{2} \leq x \leq x_{0}-a+\frac{l}{2}, \\
\rho_{i} & x_{0}-a+\frac{l}{2} \leq x \leq x_{0}+a-\frac{l}{2}, \\
f_{2}(x) & x_{0}+a-\frac{l}{2} \leq x \leq x_{0}+a+\frac{l}{2}, \\
\rho_{\mathrm{e}} & x \geq x_{0}+a+\frac{l}{2},
\end{array}\right\}
$$

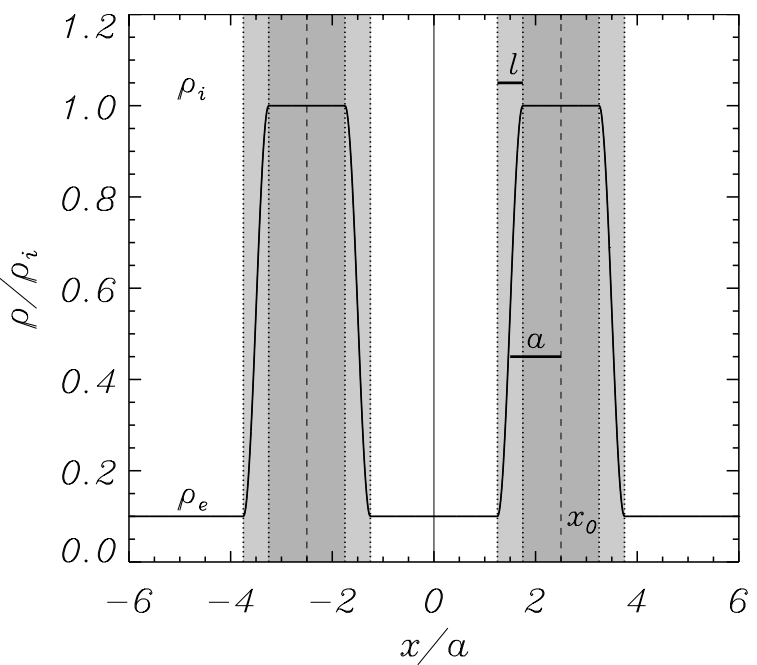

Fig. 1. Schematic representation of the two density enhancements (shaded regions) of half-width $a$, centred on $\pm x_{0}$ and representing a coronal loop composed of two slabs in the direction transverse to the equilibrium magnetic field. These enhancements with internal density $\rho_{\mathrm{i}}$ connect to the external medium, with density $\rho_{\mathrm{e}}$, by transitional nonuniform layers (light-shaded regions) of thickness $l$.

with $\rho(-x)=\rho(x)$. The density profiles at the non-uniform transitional layers have been chosen following Ruderman \& Roberts (2002) and Van Doorsselaere et al. (2004) and are given by

$f_{1}(x)=\frac{\rho_{i}}{2}\left[\left(1+\frac{\rho_{\mathrm{e}}}{\rho_{i}}\right)-\left(1-\frac{\rho_{\mathrm{e}}}{\rho_{i}}\right) \sin \frac{\pi\left[-x-\left(-x_{0}+a\right)\right]}{l}\right]$

and

$f_{2}(x)=\frac{\rho_{i}}{2}\left[\left(1+\frac{\rho_{\mathrm{e}}}{\rho_{i}}\right)-\left(1-\frac{\rho_{\mathrm{e}}}{\rho_{i}}\right) \sin \frac{\pi\left[x-\left(x_{0}+a\right)\right]}{l}\right]$.

It is important to point out that the particular form of the density profile is not important for the results.

In this paper, the normal oscillation modes of this two-slab system will be compared to two similar physical models for a coronal loop without internal density structuring. We have considered: (1) a single equivalent slab with the same mass, width $2 a_{\mathrm{s}}$, internal density

$\rho_{\mathrm{m}}=\frac{2 a-\frac{l}{2}}{x_{0}+a} \rho_{i}+\frac{x_{0}-a+\frac{l}{2}}{x_{0}+a} \rho_{\mathrm{e}}$

and similar external non-uniform transitional layers as the twoslab system; and (2) a single slab with width $2 a_{\mathrm{s}}$, the same internal density and external layers as the two-slab system, and therefore, a different total mass. The corresponding density profiles for the three physical systems are depicted in Fig. 2. This figure shows three configurations with the density profiles, corresponding to the considered physical systems for three different values of the separation between the slabs, going from no separation (Fig. 2a) to a small separation (Fig. 2c). In the first situation (Fig. 2a), the three physical systems totally coincide. As the distance between the slabs increases, more resonance layers should be available at the new non-uniform internal transitional layers, which could lead to significant differences between the properties of the eigenmodes of the three systems. 

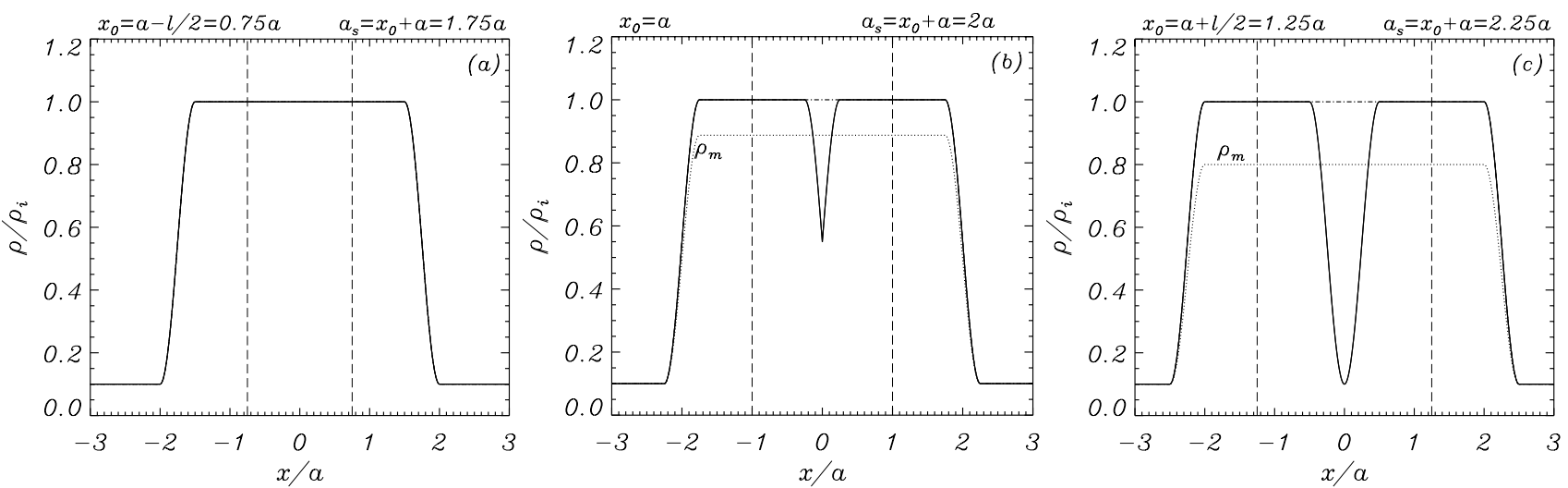

Fig. 2. Schematic representation of the density profile of two slabs (solid), one slab with same density and half-width, $a_{\mathrm{s}}$ (dash-dotted) and one slab with same total mass and half-width $a_{\mathrm{s}}$ and with weighted internal density $\rho_{\mathrm{m}}$ (dotted). Three values of the slab separation ( $2 x_{0}=$ distance between vertical dashed lines) are shown, varying from no separation, a): $x_{0}=a-l / 2$, to small separation, $\mathbf{c}$ ): $x_{0}=a+l / 2$, simulating a coronal loop with unresolved internal structure.

\subsection{Linear resistive $M H D$ waves}

To study small amplitude oscillations of the previous configuration, the resistive MHD equations with constant resistivity $\eta$ are linearised. As the equilibrium configuration only depends on the $x$-direction, a spatial and temporal dependence of the form $\exp ^{l\left(\omega t-k_{y} y-k_{z} z\right)}$ is assumed for all perturbed quantities, with $\omega=\omega_{\mathrm{R}}+\imath \omega_{\mathrm{I}}$ the complex frequency and $k_{y}$ and $k_{z}$ the perpendicular and parallel wave numbers. This leads to the following set of differential equations for the two components of the velocity perturbation, $v_{x}$ and $v_{y}$, and the three components of the perturbed magnetic field, $b_{1 x}, b_{1 y}$, and $b_{1 z}$,

$$
\begin{aligned}
\omega v_{x} & =\frac{B}{\rho}\left(-k_{z} b_{1 x}+\imath \frac{\mathrm{d} b_{1 z}}{\mathrm{~d} x}\right), \\
\omega v_{y} & =\frac{B}{\rho}\left(-k_{z} b_{1 y}+k_{y} b_{1 z}\right), \\
\omega \frac{b_{1 x}}{B} & =-k_{z} v_{x}-\imath \frac{\eta}{B}\left[\frac{\mathrm{d}^{2} b_{1 x}}{\mathrm{~d} x^{2}}-\left(k_{y}^{2}+k_{z}^{2}\right) b_{1 x}\right], \\
\omega \frac{b_{1 y}}{B} & =-k_{z} v_{y}-\imath \frac{\eta}{B}\left[\frac{\mathrm{d}^{2} b_{1 y}}{\mathrm{~d} x^{2}}-\left(k_{y}^{2}+k_{z}^{2}\right) b_{1 y}\right], \\
\omega \frac{b_{1 z}}{B} & =\left(l \frac{\mathrm{d} v_{x}}{\mathrm{~d} x}+k_{y} v_{y}\right)-\imath \frac{\eta}{B}\left[\frac{\mathrm{d}^{2} b_{1 z}}{\mathrm{~d} x^{2}}-\left(k_{y}^{2}+k_{z}^{2}\right) b_{1 z}\right] .
\end{aligned}
$$

When $k_{y} \neq 0$, Eqs. (5)-(9) define an eigenvalue problem for coupled fast and Alfvén normal modes. As the plasma- $\beta=0$, the slow mode is absent and there are no motions parallel to the equilibrium magnetic field, $v_{z}=0$. Solutions to these equations are usually difficult to obtain, when perpendicular propagation $\left(k_{y} \neq 0\right)$ is considered and transitional non-uniform layers $(l \neq 0)$ are included. For this reason numerical approximations to the solutions are obtained for constant resistivity and for a given density profile $\rho(x)$. The numerical solutions have been obtained using PDE2D (Sewell 2005), a general-purpose partial differential equation solver. The numerical code uses finite elements and allows the use of a non-uniformly distributed grid, in order to better resolve the steep gradients that arise in the vicinity of the resonant layers. An important point from a practical point of view is that resistivity has to be small enough for the imaginary part of the frequency of resonantly damped fast modes to be independent of resistivity (Poedts \& Kerner 1991). This condition has been checked for the fundamental kink mode of oscillation of the two-slab system for a given range of values for the

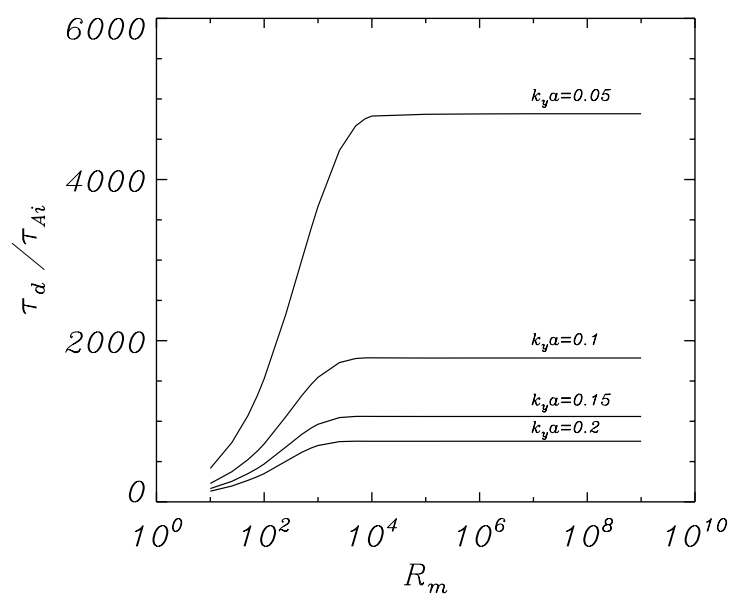

Fig. 3. Damping time (in units of the internal Alfvén transit time, $\left.\tau_{\mathrm{A}}=a / v_{\mathrm{Ai}}\right)$ vs. the magnetic Reynolds number, $R_{\mathrm{m}}=v_{\mathrm{Ai}} a / \eta$, with $\eta$ the resistivity, for the fundamental symmetric mode and four different values of the perpendicular propagation in a two-slab system with $x_{0} / a=2.25$. For values of the Reynolds number in the range $\left[10^{4}, 10^{9}\right]$ the damping time is independent of diffusion, such as is required by the correspondence between the resistive eigenmode and its ideal counterpart or quasi-mode (Poedts \& Kerner 1991). The equilibrium parameters are $k_{z} a=\pi / 50, \rho_{\mathrm{i}} / \rho_{\mathrm{e}}=10$, and $l / a=0.5$.

thickness of the non-uniform layers, $l$, and perpendicular wave number, $k_{y}$. Figure 3 displays the dependence of the damping time obtained for a given value of $l$ and different values of $k_{y}$. One can appreciate that a value of $R_{\mathrm{m}}=10^{8}$ is high enough to achieve the required independence of the damping time on the magnetic Reynolds number. By increasing the value of $R_{\mathrm{m}}$, the solution becomes more "singular" at the resonant layers, hence the number of grid points have to be chosen so as to be able to resolve the solution at those locations. The use of a 1D model turns out to be of particular interest, since a relatively small number of grid points has been sufficient for obtaining all the numerical solutions, at a reasonable computational cost. Finally, regarding the boundary conditions, we impose the vanishing of the perturbed velocity far away from the loop, hence $\boldsymbol{v}=\mathbf{0}$ at $x \rightarrow \pm \infty$.

\section{Analysis and results}

The fast normal oscillation modes for a system of two coronal loops, modelled in slab geometry, have been studied recently by 

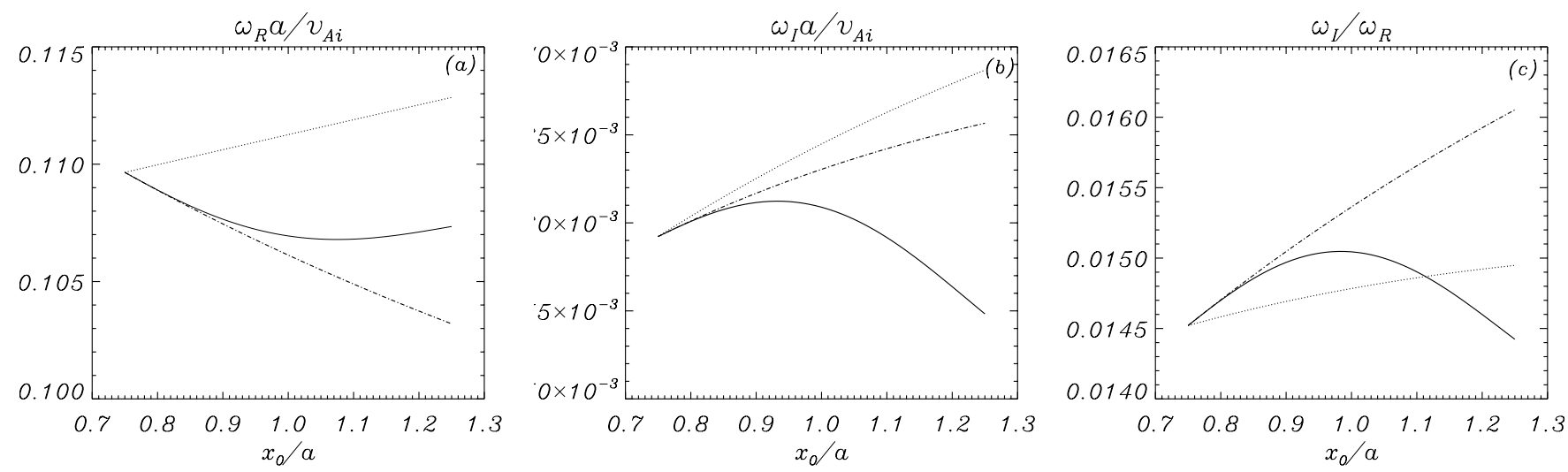

Fig. 4. Real part of the frequency a), imaginary part of the frequency b) and damping rate c), as a function of half the slab separation in units of $a$ for a system of two slabs (solid line), a single equivalent slab with equal mass and width (dotted-line), and single equivalent slab with equal density contrast and width (dash-dotted line).

Luna et al. (2006) in the absence of perpendicular propagation $\left(k_{y}=0\right)$ and for piecewise constant density profiles $(l=0)$. These authors find that the system is able to support a variety of oscillatory modes. In particular, the fundamental mode is symmetric with respect to $x=0$, with the two slabs oscillating in phase with the same frequency. The frequency of this eigenmode is constrained between two limiting values. In the limit of no separation, it is equal to the frequency of the kink mode of a single slab with double width. As the distance between the slabs increases, the interaction weakens and, for large separation, the two slabs do not "feel" each other anymore and the oscillatory frequency of the whole system is the same as the kink mode frequency of a single slab. There is also an antisymmetric mode; in this case, the two slabs oscillate with the same frequency, but in opposition of phase. Luna et al. (2006) find that, although the symmetric mode is present for all possible values of the distance between the slabs, the same is not true for the antisymmetric mode, which becomes leaky when the distance between the slabs is smaller than a certain value, which depends on the value of the longitudinal wave number. In this paper, we only consider the fundamental symmetric kink mode of oscillation of the two-slab system. The general oscillatory properties of a system of two coronal loops with perpendicular propagation and with non-uniform layers for different equilibrium parameters will be presented elsewhere.

When compared to Luna et al. (2006), there are two new ingredients in the physical model under consideration in this paper. On one hand, the equilibrium density is non-uniform at the edges of the slabs. On the other, perpendicular propagation of the perturbations is included. When perturbations are allowed to propagate in the perpendicular direction $\left(k_{y} \neq 0\right)$, the collective fast mode is coupled to Alfvén modes. If, in addition, the density is allowed to vary in a non-uniform transitional layer, resonant coupling between the fast mode and Alfvén continuum modes takes place and, as a result, the global mode of oscillation is damped. The real part of the frequency gives the period of the oscillation, $P=2 \pi / \omega_{\mathrm{R}}$, while the imaginary part is related to the damping time, $\tau_{\mathrm{d}}=1 / \omega_{\mathrm{I}}$. In this work, the real and imaginary parts of the frequency of resonantly damped oscillations are computed.

In our numerical calculations, we consider fixed values for the density contrast of the two-slab system, $\rho_{\mathrm{i}} / \rho_{\mathrm{e}}=10$ and for the longitudinal wavenumber $k_{z} a=\pi / 50$. For the observed kink oscillation with a wave-length double the length of the loop, this corresponds to a ratio of length to width $L / 2 a=25$. These are typical values for observed coronal loops. We also consider a fixed value for the perpendicular wave number, $k_{y} a=0.2$, and the thickness of the non-uniform layer, $l / a=0.5$. Increasing the value of the former produces a decrease in the damping time, such as can be seen in Fig. 3, while the damping rate also depends on the value of the latter, such as shown by Van Doorsselaere et al. (2004). In any case, our results do not change qualitatively when different values for these quantities are taken. The distance between the centres of the slabs in the two-slab system is varied, as shown in Fig. 2, in a range going from no separation with $x_{0}=a-l / 2$ to small separation, with $x_{0}=a+l / 2$. These limits have been chosen in such a way that we have the total equivalence of the three systems for the lower limit. On the other hand, for the larger separation we considered, the total width of the system is still representative of actual coronal loops. As we increase the distance between the slabs, the weighted mean internal density, $\rho_{\mathrm{m}}$, of the single equivalent slab with the same total mass decreases and reaches $80 \%$ of $\rho_{\mathrm{i}}$.

The real and imaginary parts of the frequency for the three systems, together with the resulting damping rate, are computed and the results are displayed in Fig. 4. These figures show that the period, damping time, and damping per period of a system of two slabs vary when the distance between the two slabs is increased. As for the two equivalent single slab systems, variations in $x_{0}$ are related to variations in their half-width, given by the relation $a_{\mathrm{s}}=a+x_{0}$. These variations produce the change in the real and imaginary parts of the frequency since the total width and the mean density (in one case) are changed. This only reflects that different physical systems are considered when varying $x_{0}$. However, when the values of the period, damping time, and damping rate are considered, we see that the values corresponding to the two-slab system differ by less that $15 \%$ when compared to a single slab with the same width and either the same density or the same total mass.

In order to shed light on the interaction between the fast modes and the Alfvén continuum modes, we look at the values of the computed frequencies with respect to the frequency of the Alfvén continuum. Figures $5 \mathrm{a}-\mathrm{c}$ show the transversal profiles of the Alfvén frequency, $\omega_{\mathrm{A}}=k_{z} v_{\mathrm{A}}$, corresponding to the three configurations depicted in Figs. $2 \mathrm{a}-\mathrm{c}$ and the computed real parts of the frequency, obtained for each of the three considered physical systems. We can appreciate that, first, the differences in frequency between the three physical systems are rather small and, second, that when there is no separation between the two slabs or when this separation is relatively small, the real part of the 

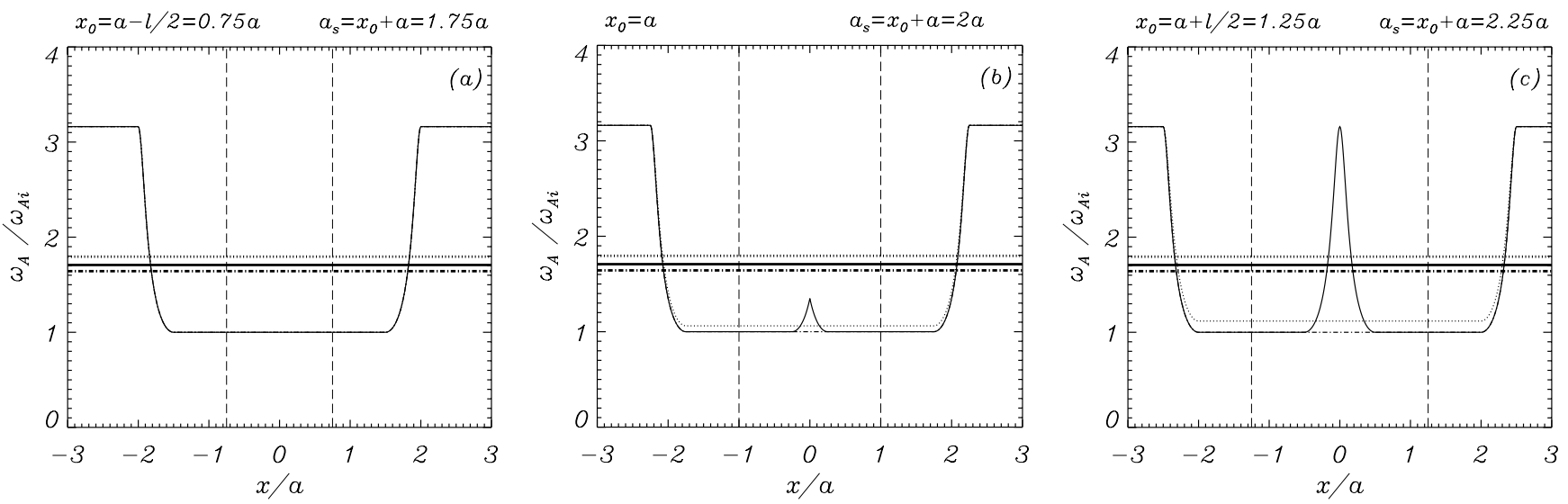

Fig. 5. Alfvén frequency profiles corresponding to the three configurations displayed in Fig. 2. The horizontal lines are the values for the real part of the frequency corresponding to the computed eigenmode for the two-slab system (solid), single equivalent slab with equal mass and width (dotted-line), and single equivalent slab with equal density contrast and width (dash-dotted line).

frequency for the eigenmodes of the three systems only has crossings with the two Alfvén continua that correspond to the external non-uniform layers of the full system. On the other hand, when the separation between the slabs is further increased (see Figs. 2c and 5c), the real part of the frequency of the two-slab system is inside the Alfvén continua corresponding to both the external and internal non-uniform layers. Although one could think that this should produce a significant difference in the damping rate of the eigenmodes under consideration, our results indicate that this is not the case and that the new available resonant couplings do indeed result in little effect on the imaginary part of the frequency (Fig. 4b) and on the corresponding damping rate (Fig. 4c). Hence, under the present circumstances, the internal structuring looks almost irrelevant.

To gain further insight into the properties of the oscillatory modes of the physical systems that are being compared and to understand the results displayed in Fig. 4 better, we now consider the spatial distribution of the eigenfunctions. They are complex quantities, as are the frequencies. Figure 6 shows the perturbed transverse velocity, $v_{x}$, and the perturbed magnetic pressure, $P_{\mathrm{T}}$ (obtained from $b_{1 z}$ ), for two different values of the separation between the slabs in the two-slab system. For these parameters and for both values of the distance, the system is allowed to have resonant couplings at the internal layers of the full system, and the real part of the frequency is inside the corresponding Alfvén continua.

Due to the resonant coupling of the fast kink mode to Alfvén modes the peaks in the perturbed velocity are clearly visible in the external non-uniform layers in Figs. 6a (slabs just touching) and $6 \mathrm{c}$. No appreciable signature of resonant coupling in the internal layers is visible in Fig. 6a. These peaks are clearly visible when the distance between the slabs is increased further, thus producing the smaller peaks in the internal layers in Fig. 6c. Regarding the perturbation to the total pressure, this function has to vanish at the centre of the system, so it does not reach high values when the two slabs are just touching (Fig. 6b). The amplitude of the perturbed total pressure is large in the internal layers when the distance between the slabs is increased, although still small when compared to its amplitude in the external layers.

Sakurai et al. (1991) in ideal MHD and Goossens et al. (1995) in dissipative MHD have computed the jumps in the normal component of the displacement and in the Eulerian perturbation of the total pressure for the driven problem in cylindrical geometry. For a straight field, they find that the total pressure is continuous and that the jump in the component of the displacement is proportional to the total pressure. These results were extended to the eigenvalue problem by Tirry \& Goossens (1996), both for cylindrical and Cartesian geometry. An expression for the jump in the energy flux across the dissipative layer has been derived by Andries et al. (2000) and Andries \& Goossens (2001). For a straight field, this jump is proportional to the total pressure squared. This result is applicable to both cylindrical and Cartesian geometry. We can therefore interpret the results displayed in Fig. 4 and the small effect of the internal density structuring of a single coronal loop as being due to the predominance of the resonant couplings in the external layers, when compared to those occurring in the internal ones, due to the relative importance of the magnitude of the perturbed magnetic pressure in the external layers with respect to its magnitude in the internal ones.

We have obtained further confirmation regarding the relative importance of the resonant couplings at the external layers with respect to the internal ones by performing another simple numerical experiment. We computed the imaginary part of the frequency of the two-slab system, as a function of the separation between the slabs, by considering, separately, their values when only the internal layers are present and when only the external ones provide the damping by resonant conversion of energy. Our results are displayed in Fig. 7. This figure indicates that the imaginary part of the frequency of the two-slab system turns out to be 3 orders of magnitude smaller when only the internal layers are present, while it is similar to the previously considered two-slab system with both internal and external layers, when only the external layers are present. We can thus conclude that the external layers are mainly responsible for the damping of the eigenmode.

\section{Summary and conclusions}

We have studied the influence of the possibly unresolved density structure of a coronal loop on the oscillatory properties of its resonantly damped transverse oscillations. We concentrated our analysis on the effect of a non-uniform radial distribution of the density, in the interior of a coronal loop, on the period of the oscillation and on the damping due to resonant coupling to Alfvén waves. To model the sub-resolution structuring of a coronal loop, a single monolithic loop was modelled as being composed of a system of two identical parallel coronal slabs. The fundamental mode of oscillation of the two-slab system turns out to be 

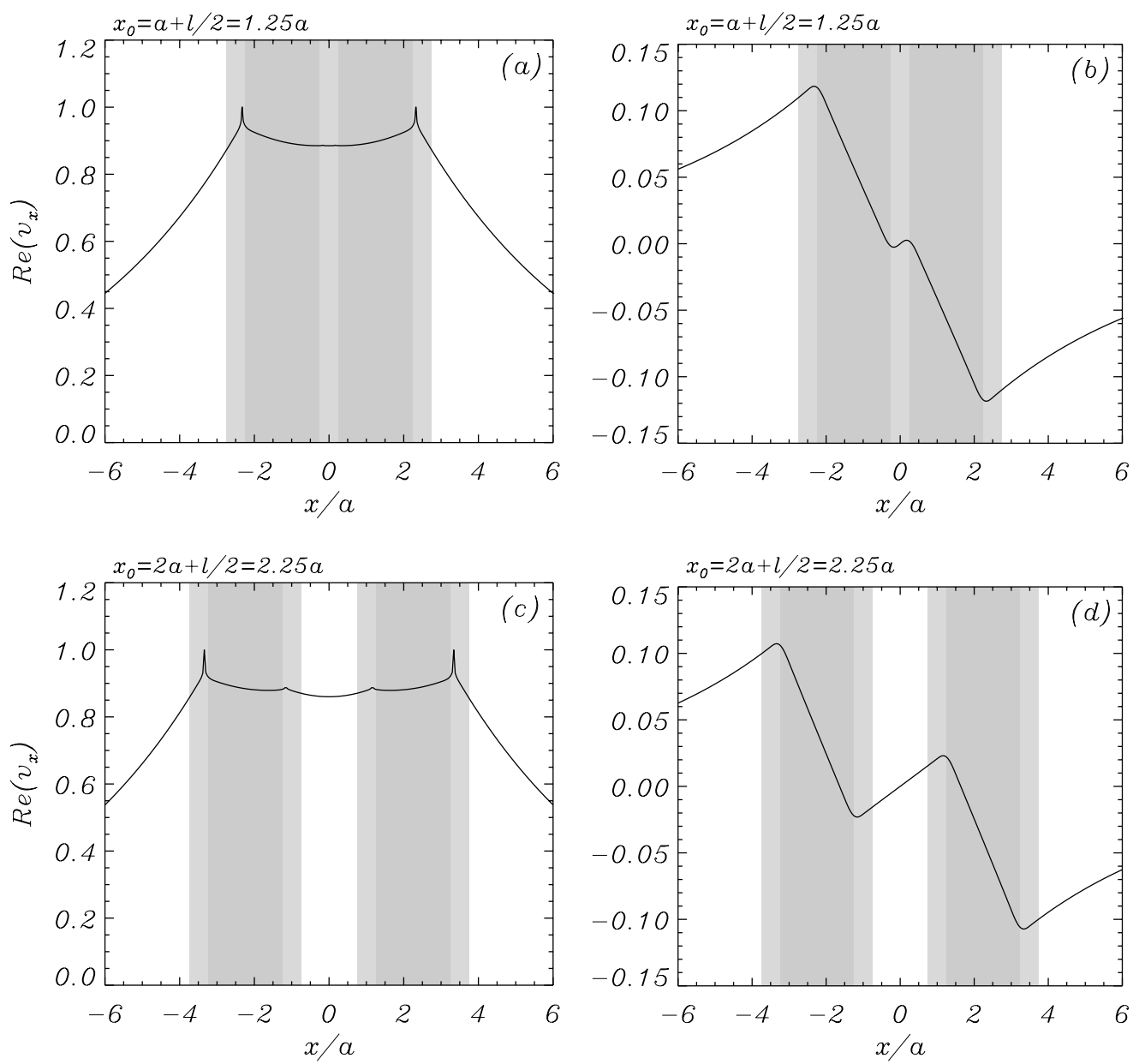

Fig. 6. a) and c): real part of the transverse velocity perturbation for the resonantly damped eigenmode with $\rho_{\mathrm{i}} / \rho_{\mathrm{e}}=10, k_{z} a=\pi / 50, k_{y} a=0.2$, and $R_{\mathrm{m}}=10^{8}$, for two different values of the distance between the two slabs, $x_{0}$. b) and d): imaginary part of the total pressure perturbation, for the same values of the distances.

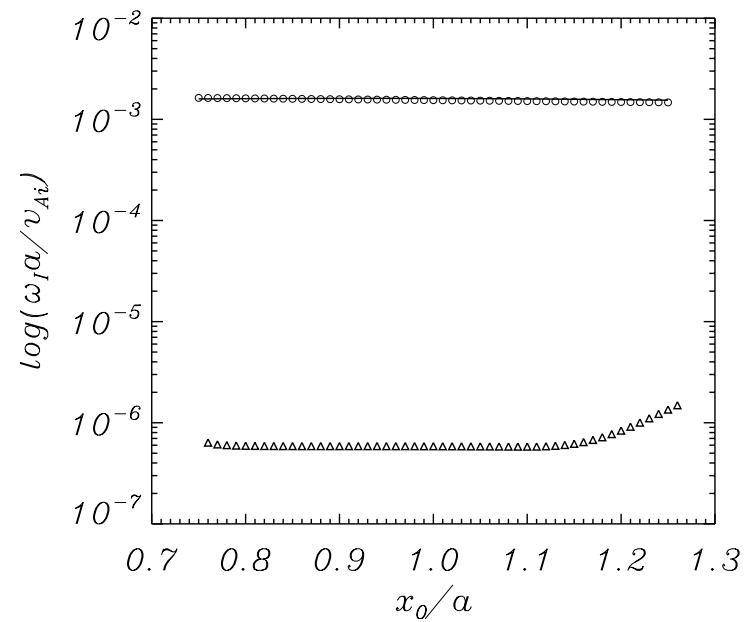

Fig. 7. Imaginary part of the frequency for the fundamental resonantly damped fast eigenmode in a two-slab configuration with either only the internal non-uniform layers (triangles) or only the external non-uniform layers (circles). The solid line indicates the solutions with both internal and external layers. The parameters are $\rho_{\mathrm{i}} / \rho_{\mathrm{e}}=10, k_{z} a=\pi / 50, k_{y} a=$ 0.2 , and $R_{\mathrm{m}}=10^{8}$.

symmetric (Luna et al. 2006) so should resemble the fast kink eigenmode of a monolithic model of coronal loop, such as is observed by TRACE. As perpendicular propagation of the perturbations is considered and the internal density on each of the slabs is connected to the coronal environment by means of non-uniform layers, these eigenmodes are resonantly damped oscillations. The real and imaginary parts of the frequency of the fundamental symmetric eigenmode have been computed for different values for the distance between the two slabs, from no separation to a small separation. For each value of the separation between the slabs, we have also computed the real and imaginary parts of the frequency of two equivalent models of coronal slabs with uniform internal density. One of these models has the same density contrast and external layers and the other keeps the same total mass while having different internal density.

When the values of the computed quantities are compared, our results indicate that the period, damping time, and damping rate of the system of two slabs change very little with respect to the same quantities for any of the two equivalent, uniform single-slab models. By studying the spatial distribution of the perturbed transverse velocity and perturbed total pressure, we find that this is due to the predominance of the resonant coupling in the external layers of the system.

Further confirmation of this result was obtained by separately computing the imaginary part of the frequency when only the internal layers of the full system or only the external ones were present. Our results indicate that the damping of the kink eigenmode is produced mainly by the resonant couplings in the external layers. We thus conclude that the influence of the internal structure of the density in a coronal loop on the features of the 
resonantly damped fast mode oscillations is not very relevant. This result can, in principle, be extended to the case where more sub-resolution density enhancements are present; i.e. more loops or more internal non-uniform layers do not necessarily mean that the damping time would be different. Our results, therefore, disagree with the claim by Ruderman \& Roberts (2002) that, when small-scale loop structures are considered, one may expect that resonant absorption will then occur not only in one but also in $n$ resonant layers, and the damping rate will be scaled accordingly by a factor of order $n l / a$. Our results also disagree with numerical computations by Ofman (2005), obtained for a bundle of four loops, for which the damping time is found to be different to the one obtained for a single loop.

Acknowledgements. The authors acknowledge the Spanish Ministerio de Educación y Ciencia for funding provided under projects AYA2003-00123 and AYA2006-07637 and the Conselleria d'Economia, Hisenda i Innovació of the Government of the Balearic Islands for the funding provided under grant PRIB2004-10145. J. Terradas thanks the Spanish Ministerio de Educación y Ciencia for funding provided under a Juan de la Cierva fellowship. The authors also thank Marcel Goossens, Jesse Andries, and Tom Van Doorsselaere for helpful conversations.

\section{References}

Andries, J., \& Goossens, M. 2001, A\&A, 368, 1083

Andries, J., Tirry, W. J., \& Goossens, M. 2000, ApJ, 531, 561

Andries, J., Arregui, I., \& Goossens, M. 2005a, ApJ, 624, L57

Andries, J., Goossens, M., Hollweg, J. V., Arregui, I., \& Van Doorsselaere, T. 2005b, A\&A, 430, 1109

Arregui, I., Van Doorsselaere, T., Andries, J., Goossens, M., \& Kimpe, D. 2005, A\&A, 441, 361

Arregui, I., Andries, J., Van Doorsselaere, T., Goossens, M., \& Poedts, S. 2007, A\&A, 463, 333

Aschwanden, M. J. 2005, ApJ, 634, L193
Aschwanden, M. J. 2006, Roy. Soc. London Philos. Trans. Ser. A, 364, 417 Aschwanden, M. J., \& Nightingale, R. W. 2005, ApJ, 633, 499

Aschwanden, M. J., Fletcher, L., Schrijver, C. J., \& Alexander, D. 1999, ApJ, 520,880

Aschwanden, M. J., De Pontieu, B., Schrijver, C. J., \& Title, A. M. 2002, Sol. Phys., 206, 99

De Pontieu, B., Martens, P. C. H., \& Hudson, H. S. 2001, ApJ, 558, 859

Edwin, P. M., \& Roberts, B. 1983, Sol. Phys., 88, 179

Goossens, M., Ruderman, M. S., \& Hollweg, J. V. 1995, Sol. Phys., 157, 75

Goossens, M., Andries, J., \& Aschwanden, M. J. 2002, A\&A, 394, L39

Goossens, M., Andries, J., \& Arregui, I. 2006, Roy. Soc. London Philos. Trans. Ser. A, 364, 433

Hollweg, J. V., \& Yang, G. 1988, J. Geophys. Res., 93, 5423

Luna, M., Terradas, J., Oliver, R., \& Ballester, J. L. 2006, A\&A, 457, 1071

Martens, P. C. H., Cirtain, J. W., \& Schmelz, J. T. 2002, ApJ, 577, L115

Nakariakov, V. M., \& Ofman, L. 2001, A\&A, 372, L53

Nakariakov, V. M., \& Verwichte, E. 2005, Liv. Rev. Sol. Phys., 2, 3

Nakariakov, V. M., Ofman, L., DeLuca, E. E., Roberts, B., \& Davila, J. M. 1999, Science, 285,862

Ofman, L. 2002, ApJ, 568, L135

Ofman, L. 2005, Adv. Space Res., 36, 1572

Poedts, S., \& Kerner, W. 1991, Phys. Rev. Lett., 66, 2871

Roberts, B. 1983, Sol. Phys., 87, 77

Roberts, B., Edwin, P. M., \& Benz, A. O. 1984, ApJ, 279, 857

Ruderman, M. S. 2003, A\&A, 409, 287

Ruderman, M. S., \& Roberts, B. 2002, ApJ, 577, 475

Sakurai, T., Goossens, M., \& Hollweg, J. V. 1991, Sol. Phys., 133, 227

Schmelz, J. T., Beene, J. E., Nasraoui, K., et al. 2003, ApJ, 599, 604

Schmelz, J. T., Nasraoui, K., Richardson, V. L., et al. 2005, ApJ, 627, L81

Schrijver, C. J., \& Brown, D. S. 2000, ApJ, 537, L69

Schrijver, C. J., Aschwanden, M. J., \& Title, A. M. 2002, Sol. Phys., 206, 69

Sewell, G. 2005, The Numerical Solution of Ordinary and Partial Differential Equations (Wiley-Interscience)

Terradas, J., Oliver, R., \& Ballester, J. L. 2006, ApJ, 650, L91

Tirry, W. J., \& Goossens, M. 1996, ApJ, 471, 501

Uchida, Y. 1970, PASJ, 22, 341

Van Doorsselaere, T., Andries, J., Poedts, S., \& Goossens, M. 2004, ApJ, 606, 1223

Verwichte, E., Foullon, C., \& Nakariakov, V. M. 2006, A\&A, 452, 615 